\title{
Gemcitabine with or without continuous infusion 5-FU in advanced pancreatic cancer: a randomised phase II trial of the Italian oncology group for clinical research (GOIRC)
}

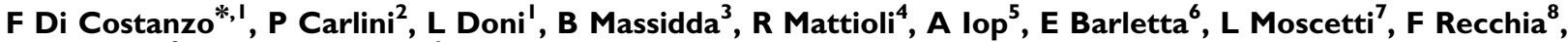 P Tralongo ${ }^{9}$ and S Gasperoni'}

'U.O di Oncologia Medica, Azienda Ospedaliera Careggi, Via Pieraccini I 7, Florence, Italy; ${ }^{2}$ U.O di Oncologia Medica A, Polo Oncologico S Raffaele Regina Elena, Roma, Italy; ${ }^{3}$ Oncologia Medica, Policlinico Universitario, Cagliari, Italy; ${ }^{4}$ U.O di Oncologia Medica, Ospedale S Croce, Fano, Italy; ${ }^{5}$ Servizio di Oncologia, Ospedale di Latisana, Udine, Italy; ${ }^{6}$ Oncologia B, Istituto Nazionale Tumori Pascale, Napoli, Italy; ${ }^{7}$ Oncologia Medica, policlinico Umberto I, Roma, Italy; ${ }^{8}$ U.O di Oncologia Medica, Ospedale di Avezzano, Italy; ${ }^{9}$ U.O di oncologia Medica, Ospedale G Di Maria, Avola, Siracusa, Italy

This study was performed to determine the activity of adding continuous infusion (Cl) of 5-fluorouracil (5-FU) to gemcitabine (GEM) vs GEM alone in advanced pancreatic cancer (APC). In all, 94 chemo-naïve patients with APC were randomised to receive GEM alone (arm A: $1000 \mathrm{mg} \mathrm{m}^{-2}$ per week for 7 weeks followed by a 2 week rest period, then weekly for 3 consecutive weeks out of every 4 weeks) or in combination with Cl 5-FU (arm B: Cl 5-FU $200 \mathrm{mg} \mathrm{m}^{-2}$ day $^{-1}$ for 6 weeks followed by a 2 week rest period, then for 3 weeks every 4 weeks). Overall response rate (RR) was the primary end point and criteria for decision were planned according to the Simon's optimal two-stage design. The overall RR was 8\% (arm A) and I I\% (arm B) (95\% confidence interval: 0.5$16 \%$ and 2-22\%), respectively, and stable disease was 29 and $28 \%$. The median duration of RR was 34 weeks (range $25-10$ I weeks) for GEM and 26 weeks (range 16-46 weeks) for the combination. The median progression-free survival (PFS) was 14 weeks (range 2-65 weeks) and 18 weeks (range 4-5I weeks), respectively. The median overall survival (OS) was 31 weeks (range I- I0I weeks) and 30 weeks $(\mathrm{I}-10 \mathrm{I}$ weeks). Toxicity was mild in both arms. This study does not show promising activity in terms of RR, PFS and OS for the double combination arm in APC.

British Journal of Cancer (2005) 93, I85- 189. doi: I0.1038/sj.bjc.6602640 www.bjcancer.com

Published online 28 June 2005

(c) 2005 Cancer Research UK

Keywords: 5-FU; gemcitabine; advanced pancreatic cancer

Pancreatic cancer is the fifth most common cancer worldwide. Unfortunately, most patients have locally advanced or metastatic pancreatic cancer and usually are poorly responsive to chemotherapy (Greenlee et al, 2001). Randomised studies have revealed a significant better overall survival (OS) with chemotherapy against best supportive care (BSC) (Weinerman and MacCormick, 1994; Glimelius et al, 1996). Although the increase in median survival was modest: 4 and 6 months for the treatment group as compared with 3 and 2.5 months for BSC, respectively, various schedules and different regimens have been evaluated with no proven benefit over single-agent 5-fluorouracil (5-FU) (Cullinan et al, 1985; Cullinan et al, 1990). In advanced pancreatic cancer (APC), continuous infusion (CI) of 5-FU is superior to 5 -FU bolus in terms of response rate (RR) and toxicity (Hansen et al, 1988; Hidalgo et al, 1999).

Gemcitabine (GEM), a deoxycitidine analogue, has demonstrated activity in APC in terms of clinical benefit and survival (Rothenberg et al, 1996). A phase III trial compared GEM vs 5-FU alone, the GEM arm showed a statistically longer survival and better clinical benefit (Burris et al, 1997). Phase I and II trials of GEM plus 5-FU bolus reported interesting results in terms of

*Correspondence: Dr Di Costanzo; E-mail: dicostanzofrancesco@tiscali.it Received 21 January 2005; revised 6 April 2005; accepted 8 April 2005; published online 28 June 2005 clinical benefit (38-66\%). Recently, Eastern Cooperative Oncology Group (ECOG) randomised patients with APC to 5-FU plus GEM $v s$ GEM alone. The OS was better, but not significant in the combination arm (6.7 vs 5.4 months; $P=0.09)$ and grade 3 and 4 leukopenia, thrombocytopenia and diarrhoea was higher in the combination arm. Several combination regimens with 5-FU, cisplatin, capecitabine, irinotecan, oxaliplatin, premetrexed and docetaxel were developed without a sure advantage (Cartwright et al, 2002; Kindler, 2002; Lutz et al, 2002; Schilsky et al, 2002; Pizzolato and Saltz, 2003; Schneider et al, 2003).

The current multicentre randomised phase II study was started to investigate the feasibility and activity of the combination with GEM plus CI 5-FU vs GEM alone in patients with APC. The rationale of the combination of 5-FU with $\mathrm{CI}$ and GEM is to prolong thymidylate synthetase inhibition, increasing the chance of pharmacodynamic with GEM and to reduce toxicity with respect to 5-FU bolus.

\section{MATERIALS AND METHODS}

\section{Patient eligibility}

The following inclusion criteria were mandatory to the study: histological or cytological diagnosis of APC (locally advanced or metastatic) with bidimensionally measurable disease. Patients were 
not amenable to surgery or radiotherapy. Karnofsky performance status (KPS) was 50 or greater, age between 18 and 75 years, no prior chemotherapy, no central nervous system metastases, life expectancy of at least 3 months, adequate haematological (neutrophil count $>1500 \mathrm{dl}^{-1}$; platelet count $>100000 \mathrm{dl}^{-1}$ ), renal (serum creatinine $<1.5 \times$ the upper limit of normal (ULN) value) and hepatic (alkaline phosphatase $<3 \times$ ULN value and bilirubin $<1.5 \times$ ULN value) functions. The protocol was approved by the Ethical Committee and written informed consent was obtained from all patients.

\section{Treatment plan}

Patients were centrally randomised by the central office of the Italian Oncology Group for Clinical Research (GOIRC) to receive: GEM alone (arm A) or in combination with CI 5-FU (arm B). GEM was administered in both arms as a 30 -min intravenous (i.v.) infusion at the dose of $1000 \mathrm{mg} \mathrm{m}^{-2}$ once per week for 7 weeks, followed by 2 weeks of rest. Thereafter, GEM was administered once weekly for 3 consecutive weeks out of every 4 weeks. In arm $\mathrm{B}, \mathrm{GEM}$ was combined with CI 5-FU $200 \mathrm{mg} \mathrm{m}^{-2}$ day $^{-1}$ i.v. for 6 weeks in the first cycle, followed by a week of rest and then for 3 weeks every 4 weeks. All patients were treated on outpatient basis.

\section{Pretreatment evaluation and assessment of efficacy}

At screening, all patients were evaluated with physical examination, complete blood count with leucocyte differential count, serum creatinine, bilirubin, transaminase, and alkaline phosphatase, serum tumour marker (CEA and Ca 19-9) levels, ECG and computed tomography (CT) scan of chest, abdomen and pelvis of all tumour sites. During the study, patients were monitored before each cycle for medical history, physical evaluation, clinical benefit, serum chemistry and tumour markers, and evaluated for toxicity. The RR (WHO) were assessed every 2 months or earlier if clinically indicated. If a response was documented, treatment continued until progression. Clinical evaluation of response was not used. Pleural effusion, ascites and hepatomegaly were not used for response assessment. In the case of grade 2 nonhaematological toxicity or greater, patients received symptomatic therapy and 5FU therapy was stopped until the toxicity ceased and the dose was reduced in the next cycle as follows: in the case of grade 2 stomatitis, diarrhoea or HFS, dose reduction was about $25 \%$ of planned dose; in the case of grade 3 stomatitis, diarrhoea or HFS, dose reduction of $5-\mathrm{FU}$ was $50 \%$; and in the case of grade 4 stomatitis, diarrhoea or HFS, 5-FU and GEM therapy was discontinued. In the case of grade 2 haematological toxicity, GEM dose was reduced by $25 \%$; in the case of grade 3, dose reduction was 50\%; patientsdiscontinued from therapy when treatment was associated with grade 4 neutropenia and infection. Patients before each cycle received a diary for evaluation of quality of life (QoL), according to Subjective Chemotherapy Impact (SCI) questionnaire (Tamburini et al, 1991). The questionnaire allowed us to assess the duration of physical and psychological discomfort of patients during the treatment. The questionnaire consisted of two items: (1) how many disturbed days did you have during the treatment?; (2) how many days do you want to cancel during the treatment? The questionnaire was answered before each cycle.

\section{Statistical considerations}

Primary end points of this study were RR. Secondary objectives were to assess the OS, safety and tolerability of the regimens. Overall survival was calculated from the first day of treatment until the date of death. Patients alive at the end of study were censored at last observation visit. For progression-free survival (PFS), the last date PFS was the date of progression or death (if dead without progression). Patients not experiencing progression or death were censored at last observation visit. Progression-free survival and OS were described according to the Kaplan-Meier method. Descriptive statistics formed the primary basis of analysis for the data collected in this study. The study was not powered for a formal comparison of efficacy outcome measures, and these were not performed. For each arm, a Simon's optimal two-stage design was chosen. According to this approach, the expected sample size is minimised for the first stage if the regimen has low activity. It was considered that the minimum $\mathrm{RR}$ had to be at least $10 \%$ and combination would had been investigated further if it showed an RR of $25 \%$ or more. Alpha and beta errors were fixed at 5 and $20 \%$, respectively. According to these assumptions, 18 patients per arm had to be recruited in the first step if at least two responses were observed, then a further 25 patients would have been enrolled, up to a total of 43 patients per arm. A minimum of eight responses was defined in order to claim activity.

\section{RESULTS}

\section{Patient demographics}

After 30 months of accrual, 94 patients were enrolled into this trial and their characteristics are listed in Table 1. Three patients were ineligible due to nonpathologic diagnosis of APC $(n=1)$, synchronous second primary tumour $(n=1)$ and nonmeasurable disease: pleural effusion $(n=1)$. The two arms of treatment were well balanced for prognostic factors. The median age was 63 years (range: $34-75$ years). The KPS was $\geqslant 80$ in 62 patients $(68 \%)$ and $<80$ in 29 patients $(32 \%)$. In the GEM arm, 35 patients $(73 \%)$ had metastatic disease and 29 patients (67\%) in the combination. In all, 48 patients $(53 \%)$ had primary pancreatic tumour plus metastases in the liver.

Table I Patient characteristics

\begin{tabular}{|c|c|c|c|c|}
\hline \multirow[b]{2}{*}{ Characteristics } & \multicolumn{2}{|c|}{ GEM } & \multicolumn{2}{|c|}{ GEM+Cl 5-FU } \\
\hline & No. & (\%) & No. & (\%) \\
\hline Enrolled & 49 & & 45 & \\
\hline Evaluable & 48 & 98 & 43 & 96 \\
\hline Median age (years) (range) & $64(34-75)$ & & $62(44-75)$ & \\
\hline \multicolumn{5}{|l|}{ Gender } \\
\hline Male & 23 & 48 & 27 & 63 \\
\hline Female & 25 & 52 & 16 & 37 \\
\hline \multicolumn{5}{|l|}{ PS (Karnofsky) } \\
\hline$\geqslant 80$ & 33 & 69 & 29 & 67 \\
\hline$<80$ & 15 & 31 & 14 & 33 \\
\hline \multicolumn{5}{|l|}{ Previous surgery } \\
\hline Yes & 21 & 44 & 17 & 40 \\
\hline No & 27 & 56 & 26 & 60 \\
\hline \multicolumn{5}{|l|}{ Site of disease } \\
\hline Pancreas alone & 13 & 27 & 14 & 33 \\
\hline Pancreas+liver & 28 & 58 & 20 & 47 \\
\hline Pancreas+nodes & 6 & 13 & 6 & 14 \\
\hline Others & । & । & 3 & 7 \\
\hline \multicolumn{5}{|l|}{ Disease at presentation } \\
\hline Locally advanced & 13 & 27 & 14 & 33 \\
\hline Metastatic disease & 35 & 73 & 29 & 67 \\
\hline
\end{tabular}

GEM = gemcitabine; $\mathrm{Cl}=$ continuous infusion; 5 -FU =5-fluorouracil; $\quad \mathrm{PS}=$ performance status. 
Table 2 Grade of adverse effects by treatment group

\begin{tabular}{|c|c|c|c|c|c|c|}
\hline \multirow{2}{*}{ Type of toxicity } & \multicolumn{3}{|c|}{ GEM, $N=49$} & \multicolumn{3}{|c|}{ GEM+Cl 5-FU, $N=4 I$} \\
\hline & \multicolumn{6}{|c|}{ Grade (WHO) } \\
\hline \multicolumn{7}{|l|}{ Hematological } \\
\hline WBC & 28 (57\%) & I (2\%) & - & 17 (41\%) & I (2\%) & - \\
\hline Haemoglobin & $24(49 \%)$ & $3(6 \%)$ & - & $14(34 \%)$ & $3(7 \%)$ & - \\
\hline \multicolumn{7}{|l|}{ Nonhaematological } \\
\hline Nausea/vomiting & $21(43 \%)$ & - & - & $13(32 \%)$ & | (2\%) & - \\
\hline Mucositis & $7(14 \%)$ & - & - & $12(29 \%)$ & $2(5 \%)$ & - \\
\hline Diarrhoea & $2(4 \%)$ & - & - & $8(20 \%)$ & - & - \\
\hline Asthenia & $20(41 \%)$ & I (2\%) & - & $13(32 \%)$ & I (2\%) & - \\
\hline Fever & $9(18 \%)$ & I (2\%) & - & 7 (17\%) & $2(5 \%)$ & - \\
\hline
\end{tabular}

$\mathrm{GEM}=$ gemcitabine; $\mathrm{Cl}=$ continuous infusion; 5 - $\mathrm{FU}=5$-fluorouracil.

\section{Toxicity}

A total of 253 cycles of chemotherapy were administered and the median number of cycles was 2 (range: $1-6$ ). In all, 90 patients in both arms were evaluable for toxicity. The data toxicity of four patients were missing. The most common side effects are shown in Table 2. Both chemotherapy regimens were well tolerated. The principal differences between the two regimens were grade 1 and 2 mucositis (arm A/B: 14 vs 29\%) and diarrhoea (4 vs 20\%). One patient in the combination arm had grade 4 thrombocytopenia. There were no treatment-related deaths.

\section{Tumour response, PFS and survival}

In total, 91 patients were evaluable for response: 48 (98\%) and 43 (96\%) patients in arms $A$ and B, respectively. Two RR were obtained in the first stage of study according to statistical considerations. The OR rate was $9.9 \%$, with a total of nine partial responses (Table 3). There were no complete responses observed in this study. In the patients treated with GEM alone, the overall RR was $8 \%$ (95\% confidence interval, $0.5-16 \%)$, while in the combination arm, it was $11 \%$ (95\% confidence interval, $2-22 \%)$. The median duration of response was 34 weeks for GEM and 26 weeks for CI 5-FU + GEM. The median PFS was 14 weeks (range: 2-65 weeks) in the GEM arm alone and 18 weeks (range: 4-51 weeks) in the combination arm. Treatment with CI 5-FU plus GEM obtained a median OS of 31 weeks and 30 weeks in GEM alone (Figure 1). In all, 18\% (arm A) and 20\% (arm B) of patients survived over 12 months. Patients were evaluated before each cycle with QoL by SCI questionnaire. The analysis of the first cycles of treatment showed no significant increase of mean disturbed days (6.3 vs 4.8) and mean of days that patients would like to cancel during the treatment $(4.2$ vs 3.0) in the GEM alone and recombination arm. In the second cycle, the mean of disturbed days was $4.2 v s 3.0$ and cancelled days $0.2 v s$ 1.2, respectively.

\section{DISCUSSION}

Despite an increasing understanding of biology, diagnostic technology and introduction of new drugs, the OS of patients with APC remains poor. Until recently, 5-FU was considered one of the standard agents in the treatment of APC and improved therapeutic index was obtained using CI schedule.

Single-agent GEM is the currently recommended first-line treatment for APC with an RR of 10\% (range 5.4-14.3\%), median survival of 4.5 months and 1-year survival of $15-18 \%$.
Table 3 Objective response rate, duration of response, PFS and survival data

\begin{tabular}{|c|c|c|}
\hline Variable & GEM, $n$ (\%) & GEM+CI 5-FU, n (\%) \\
\hline Evaluable patients & 48 & 43 \\
\hline$C R$ & - & - \\
\hline PR & $4(8 \%)$ & $5(11 \%)$ \\
\hline ( $95 \%$ confidence interval) & $(0.5-16 \%)$ & $(2-22 \%)$ \\
\hline SD & 4 (29\%) & $12(28 \%)$ \\
\hline$P R+S D$ & $18(37 \%)$ & $17(39 \%)$ \\
\hline PD & $30(63 \%)$ & $26(61 \%)$ \\
\hline Median duration response (range) & 34 weeks $(25-101)$ & 26 weeks $(16-46)$ \\
\hline Median PFS (range) & 14 weeks $(2-65)$ & 18 weeks $(4-5 \mid)$ \\
\hline Median OS (range) & 31 weeks $(|-| 0 \mid)$ & 30 weeks $(|-10|)$ \\
\hline
\end{tabular}

$\mathrm{PFS}=$ progression free survival; $\mathrm{CR}=$ complete response; $\mathrm{PR}=$ partial response; $\mathrm{SD}=$ stable disease $\mathrm{PD}=$ progression disease.

In a phase III trial, which compared 5-FU bolus with GEM, survival (18 vs $2 \%$ at 1 year) and clinical benefit response (24 and $4.4 \%$ ) were improved in the GEM arm. In a compassionate trial of 3023 patients treated with GEM, Storniolo et al (1999) reported a median survival of 5.1 months in chemo-naïve and 3.9 in previously treated patients.

In vitro studies, in HT-29 colon cancer cells, revealed synergy when 5-FU was administered prior to GEM but not with concurrent administration (Shulz et al, 1998; Madajewicz et al, 2000). GEM depletes cellular deoxyuridine monophosphate (dUMP) pools, thereby decreasing competition with 5FdUMP at the target enzyme thymidylate synthase (TS). GEM may also inhibit TS. Finally, 5-FU metabolites may inhibit deoxycytidine monophosphate deaminase, an enzyme responsible for the inactivation of GEM monophosphate (Heinemann, 2002; GEMbased combination treatment of pancreatic cancer). Preclinical data do not evaluate the different methods of 5-FU administration such as protracted CI therapy vs bolus therapy. 5-FU bolus have reported disappointing results with a median survival of less than 5 months. More prolonged infusion schedules are associated with a median OS time of 6-8 months

The combination of GEM and 5-FU have been used in a great variety of schedules: 5-FU bolus, $\mathrm{CI}$, standard-dose 5-FU or protracted $(24-48 \mathrm{~h})$ infusion of high-dose 5 -FU given either at weekly or biweekly intervals (Castellano et al, 2000; Louvet et al, 2001; Maurel et al, 2001).

Six studies, including 164 patients with APC, have evaluated CI 5-FU (200-250 $\left.\mathrm{mg} \mathrm{m}^{-2} \mathrm{day}^{-1}\right)$ in combination with GEM in APC 


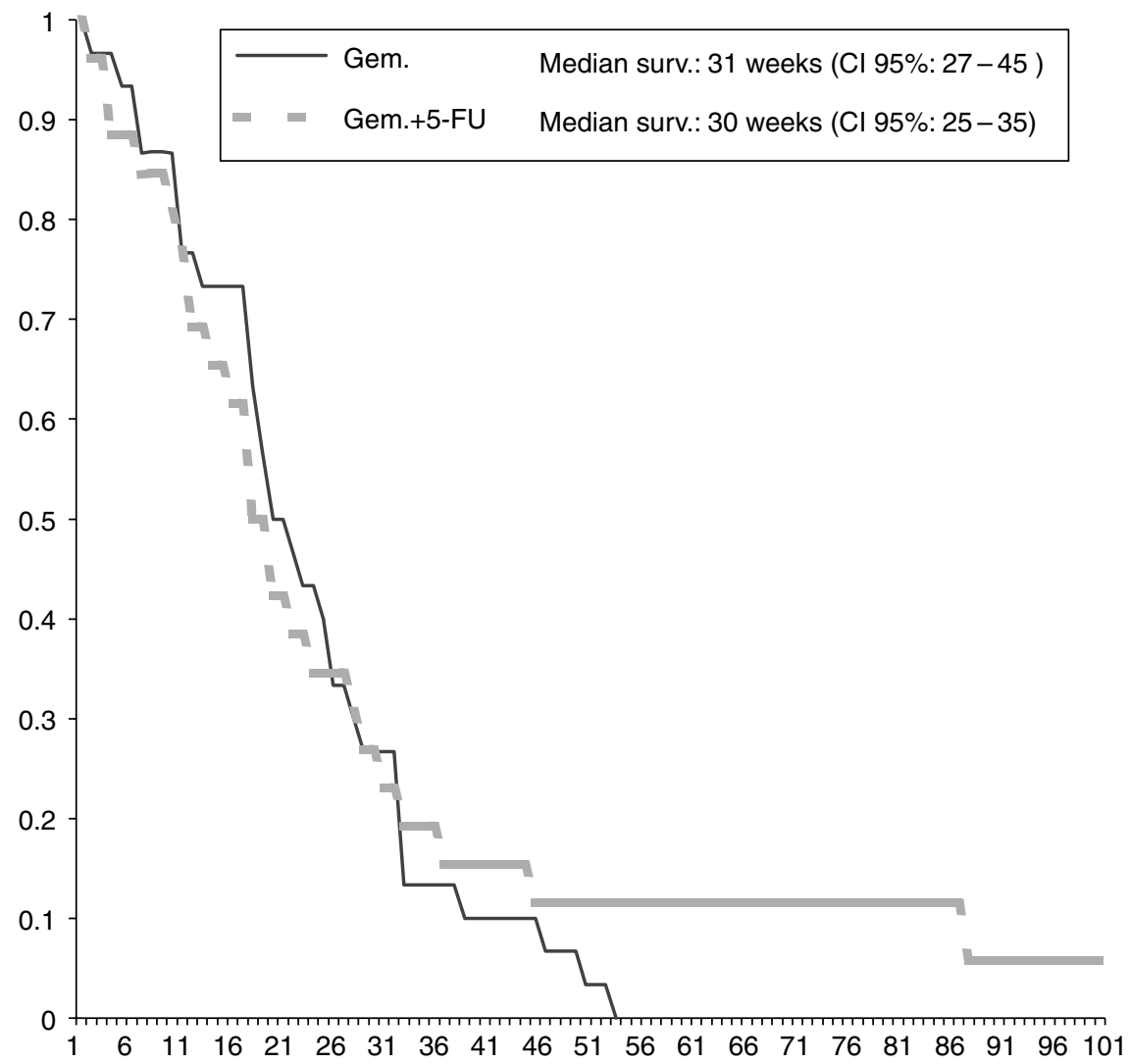

Figure I Survival curves estimated by the Kaplan-Meier method: $Y$-axis, probability of survival; $X$-axis, weeks.

(Hidalgo et al, 1999; Anchisi et al, 2000; Shulman et al, 2000 Alabiso et al, 2001; Rauch et al, 2001; Reni et al, 2001). Response rate was between 13 and $51 \%$, and OS between 5.3 and 10.3 months. Clinical benefit was only determined in three trials with an RR of 31, 45 and 78\% (Hidalgo et al, 1999; Alabiso et al, 2001; Reni et al, 2001). Kurtz et al (2000), using GEM plus CI 5-FU, reported an RR of 10 and $42 \%$ of stable disease (SD) with a median survival of 4 months. GEM plus CI 5-FU was well tolerated and major toxicities were principally mucositis, nausea/vomiting and leukopenia. The toxicities of CI 5-FU and GEM do not overlap, they can be used in combination at full doses, as demonstrated by Hidalgo et al (1999). Recent data from studies comparing GEM with or without 5 -FU suggest that 5-FU bolus administration may be of little or no benefit.

Berlin et al in a randomised trial of 327 patients used GEM alone weekly for 3 weeks out of every 4 weeks or GEM followed by 5-FU weekly on the same schedule (Berlin et al, 2002). The combination arm did not improve the median OS (6.7 vs 5.4 months) compared with GEM alone. The RR were in the range of 5.6 and $6.9 \%$ and PFS 2.2 months compared with 3.4 months for the combination. This study did not demonstrate a synergistic activity of this combination with respect to GEM alone. This trial demonstrated that GEM plus 5-FU is a tolerable regimen. Although toxicities were more common on the GEM plus 5-FU arm, there was no significant difference between the two arms. The most common grade 3 and 4 side effects were leukopenia, thrombocytopenia and diarrhoea. Other trials using 5-FU bolus plus GEM reported a median response of $14 \%$ (range $3.7-20 \%$ ) and a median survival of 6.4 months (range 4.4-7.5 months) (Gutzler et al, 1999; Castellano et al, 2000).

Our research group investigated a combination regimen of GEM plus CI 5-FU vs GEM alone in a randomised phase II trial in APC. The results do not suggest a better activity of combination over
GEM alone. The overall RR for all patients treated was $8 \%$ in GEM alone and $11 \%$ in the combination arm. The evaluation of tumour areas in pancreatic cancer is difficult, even with newer imaging techniques, because of a vigorous desmoplastic reaction, including inflammation and fibrosis within and around the tumour. In the locally advanced pancreatic cancer, we used CT scan to parameter the lesion with two perpendicular diameters, possibly excluding desmoplastic reaction even if there were possible difficulties in accuracy. In this trial, we did not obtain any objective response in the primary tumour. Finally, median TTP and OS are similar in both arms.

Historical comparisons between clinical trial are hazardous because there is considerable variability between study population, schedule and methodology of response evaluation, although with these considerations in mind the median survival for GEM alone, on GOIRC trial, was better than the principal studies with GEM alone (7 vs 5.7 months). The RR and OS in trials with GEM plus 5FU bolus ranged from 3.7 to $20 \%$ and 5-10 months vs $13-25 \%$ and 4-7 months with GEM plus CI 5-FU, respectively.

The incidence of gastrointestinal grade 3 and 4 toxicities was low in both arms. Haematological toxicity was principally grades 1 and 2 ; only one patient (2\%) developed severe thrombocytopenia. Clinical benefit response, as introduced by Burris, was not measured prospectively in this study. In this trial, patients with disease confined to the pancreas alone $(33 \%$ in the combination arm and $27 \%$ in GEM alone) did not receive radiotherapy. Although the more recent studies suggest a reasonable survival time with $\mathrm{CT}+\mathrm{RT}$, the results are not convincingly better than chemotherapy alone. Thus, it follows that it is not clear whether $\mathrm{CT}+\mathrm{RT}$ confers any survival advantage when compared with CT alone (Neoptolemos et al, 2003). The patients were evaluated for QoL by SCI questionnaire and there were no statistical differences between the two arms, although only a small number of patients 
received an evaluation for QoL after second cycles due to progression of disease.

In conclusion, treatment of APC remains a challenge. This trial demonstrated that the addition of CI 5-FU to GEM does not show sufficient activity in order to be further tested in phase III trials.
Other trials with several different schedules or modulations of 5-FU do not improve the outcome of pancreatic cancer. A future goal could be represented by the introduction of targeted therapies to improve disease control without significant added toxicity.

\section{REFERENCES}

Alabiso O, Buosi R, Clerico M, Dogliotti L, Forti L, Lattuada S, Merlano M, Ostellino O, Satolli M, Alabiso I, Sponghini A (2001) Preliminary results of a phase II study with gemcitabine and continuous infusion 5-FU in patients with advanced or metastatic pancreatic cancer. Proc Am Soc Clin Oncol 20: A2331

Anchisi S, Delaloye B, Petite J, Laurencet FL, Ambrod CH, Obrist R (2000) Gemcitabine (GEM) and continuous infusional 5-FU (Cif) is active and well tolerated in advanced or metastatic pancreatic cancer. Proc Am Soc Clin Oncol 19: A1280H

Berlin JD, Catalano P, Thomas JP, Kugler JW, Haller DG, Benson 3rd AB (2002) Phase III study of gemcitabine in combination with fluorouracil vs gemcitabine alone in patients with advanced pancreatic carcinoma: Eastern Cooperative Oncology Group Trial E2297. J Clin Oncol 20: $3270-3275$

Burris III HA, Moore MJ, Andersen J, Green MR, Rothenberg ML, Modiano MR, Cripps MC, Portenoy RK, Storniolo AM, Tarassoff P, Nelson R, Dorr FA, Stephens CD, Von Hoff DD (1997) Improvements in survival and clinical benefit with GEM as first-line therapy for patients with advanced pancreas cancer: a randomized trial. J Clin Oncol 15: 2403-2413

Cartwright TH, Cohn A, Varkey JA, Chen YM, Szatrowski TP, Cox JV, Schulz JJ (2002) Phase II study of oral capecitabine in patients with advanced or metastatic pancreatic cancer. J Clin Oncol 20: 160-164

Castellano D, Paz-Ares L, Pronk L, Tornamira MV, Tabernero J, Ciruelos E, Carcas A, Frias J, Cortes-Funes H (2000) A phase I/II clinical and pharmacologic study of dose-escalating and dose-sequencing of administration of gemcitabine (G) and folinic acid (FA)/fluorouracil (FU) in advanced pancreatic cancer (APC). Proc Am Soc Clin Oncol 19: A1133

Cullinan S, Moertel CG, Wieand HS, Schutt AJ, Krook JE, Foley JF, Norris BD, Kardinal CG, Tschetter LK, Barlow JF (1990) A phase III trial on the therapy of advanced pancreatic carcinoma: evaluations of the Mallinson regimen and combined 5-fluorouracil, doxorubicin, and cisplatin. Cancer 65: $2207-2212$

Cullinan SA, Moertel CG, Fleming TR, Rubin JR, Krook JE, Everson LK, Windschitl HE, Twito DI, Warschke RF, Foley JF (1985) A comparison of three chemotherapeutic regimens in the treatment of advanced pancreatic and gastric carcinoma: fluorouracil $v s$ fluorouracil and doxorubicin vs fluorouracil, doxorubicin, and mitomycin. JAMA 253: $2061-2067$

Glimelius B, Hoffman K, Sjoden PO, Jacobsson G, Sellstrom H, Enander LK, Linne T, Svensson C (1996) Chemotherapy improves survival and quality of life in advanced pancreatic and biliary cancer. Ann Oncol 7(6): 593-600

Greenlee RT, Hill-Harmon MB, Murray T, Thun M (2001) Cancer statistics. CA Cancer J Clin 51: 15-36

Gutzler F, Moehler M, Hosch WP, Wagner V, Nehme M, Rudi J, Stremmel W (1999) A phase I study of gemcitabine (GEM) in combination with five days 5-fluorouracil (5-FU) and folinic acid (FA) in patients with advanced adenocarcinoma of pancreas or bile duct. Proc Am Soc Clin Oncol 18: A1097

Hansen R, Quebbman E, Ritch P, Chitambar C, Anderson T (1988) Continuous 5-fluorouracil infusion in carcinoma of the pancreas. Am J Med Sci 295: $91-93$

Heinemann V (2002) Gemcitabine-based combination treatment of pancreatic cancer. Semin Oncol 29(Suppl 3): 25-35

Hidalgo M, Castellano D, Paz-Ares L, Gravalos C, Diaz-Puente M, Hitt R, Alonso S, Cortes-Funes H (1999) Phase I-II study of gemcitabine and fluorouracil as a continuous infusion in patients with pancreatic cancer. J Clin Oncol 17: 585-592

Kindler HL (2002) The pemetrexed/gemcitabine combination in pancreatic cancer. Cancer 15: $928-932$

Kurtz JE, Kohser F, Negrier S, Trillet-Lenoir V, Walters S, Limacher JM, Untereiner M, Kayitalire L, Jaeck D, Dufour P (2000) Gemcitabine and protracted 5-FU for advanced pancreatic cancer. A phase II study. Hepatogastroenterology 47(35): $1450-1453$
Louvet C, Andre T, Hammel P (2001) Phase II trial of bimonthly leucovorin, 5-fluorouracil and gemcitabine for advanced pancreatic adenocarcinoma (FOLFUGEM). Ann Oncol 12: 675-679

Lutz MP, Ducreux M, Wagener T, Van Laethem J-L, Vanhoefer U, Wils J, Gamelin E, Koene C-H, Arnaud JP, Mitry E, Husseini F, Reichardt P, El-Serafi M, Etienne P, Fleeth J, Lingenfelser T, Praet M, Baron B, Wilke H, Nordlinger B, Van Cutsem E (2002) Docetaxel/gemcitabine or docetaxel/cisplatin in advanced pancreatic carcinoma: a randomized phase II study of the EORTC-GI group. Proc Am Soc Clin Oncol 21: A498

Madajewicz S, Hentschel P, Burns P, Caruso R, Fiore J, Fried M, Malhotra H, Ostrow S, Sugarman S, Viola M (2000) Phase I chemotherapy study of biochemical modulation of folinic acid and fluorouracil by gemcitabine in patients with solid tumor malignancies. J Clin Oncol 15: 3553-3557

Maurel J, García López JL, Léon A, Bréton J, Fernandez Martos C, Florian J, Sanchez Rovira P, Casinello J, Galan M, Aranda E, Llanos M, Sastre J, Dorta J, Carrato A (2001) Phase II trial of gemcitabine (GEM) and 48-h continuous infusion (CI) of 5-fluorouracil (5-FU) as first-line treatment for patients with locally advanced and metastatic exocrine pancreatic cancer (PC). Proc Am Soc Clin Oncol 20: A2290

Neoptolemos JP, D Cunningham D, Friess H, Bassi C, Stocken DD, Tait DM, Dunn JA, Dervenis C, Lacaine F, Hickey H, Raraty MG, Ghaneh P, Buchler MW (2003) Adjuvant therapy in pancreatic cancer: historical and current perspectives. Ann Oncol 14: 675-692

Pizzolato JF, Saltz LB (2003) Irinotecan in the treatment of pancreatic cancer. Expert Rev Anticancer Ther 3: 587-593

Rauch DP, Maurer CA, Aebi S, Pampallon S, Friess H, Ludwig CU, Buchler MW, Borner MM (2001) Activity of gemcitabine and continuous infusion fluorouracil in advanced pancreatic cancer. Oncology 60: 43-48

Reni M, Passoni P, Panucci MG, Nicoletti R, Galli L, Balzano G, Zerbi A, Di Carlo V, Villa E (2001) Definitive results of a phase II trial of cisplatin, epirubicin, continuous infusion 5-fluorouracil, and gemcitabine in stage IV pancreatic adenocarcinoma. J Clin Oncol 19: 2679-2686

Rothenberg ML, Moore MJ, Cripps MC, Andersen JS, Portenoy RK, Burris 3rd HA, Green MR, Tarassoff PG, Brown TD, Casper ES, Storniolo AM, Von Hoff DD (1996) A phase II trial of GEM in patients with 5-FUrefractory pancreas cancer. Ann Oncol 7: 347-353

Schilsky RL, Bertucci D, Vogelzang NJ, Kindler HL, Ratain MJ (2002) Doseescalating study of capecitabine plus gemcitabine combination therapy in patients with advanced cancer. J Clin Oncol 20: 582-587

Schneider BP, Ganjoo KN, Seitz DE, Picus J, Fata F, Stoner C, Calley C, Loehrer PJ (2003) Phase II study of gemcitabine plus docetaxel in advanced pancreatic cancer: a Hoosier Oncology Group study. Oncology 65(3): $218-223$

Shulman KL, Kindler HL, Lad TE, Reilly K, Man S, Vokes E (2000) Phase II study of gemcitabine $(G)$ and continuous intravenous infusion (CIV) 5fluorouracil (5-FU) in advanced pancreatic cancer: a University of Chicago Phase II Consortium study. Proc Am Soc Clin Oncol 19: A1126

Shulz L, Schalhorm A, Williams W, Heinemann V (1998) Synergistic interaction of gemcitabine and 5-fluorouracil in colon cancer cells. Am Soc Clin Oncol 17: A965

Storniolo AM, Enas NH, Brown CA, Voi M, Rothenberg ML, Schilsky R (1999) An investigational new drug treatment program for patients with gemcitabine: results for over 3000 patients with pancreatic carcinoma. Cancer 85(6): $1261-1268$

Tamburini M, Brambilla C, Ferrari L, Bombino T, Gangler L, Rosso S (1991) Two simple indexes used to evaluate the impact of therapy on the quality of life of patients receiving chemotherapy for operable breast cancer. Ann Oncol 2: 417-422

Weinerman BH, MacCormick RE (1994) A phase II survival comparison of patients with adenocarcinoma of the pancreas treated with 5-fluorouracil and calcium leucovorin $v s$ a matched tumor registry control population. Am J Clin Oncol 17(6): 467-469 